\title{
Ophthalmologische Gresellschaft in Wien
}

Sitzung am 27. Mai 1929

1. Demonstrationen.

F. Fischer: Ein angeborenes Lidkolobom. Ein fünf Tage altei» Säugling, der sonst keinerlei Fehlbildungen hat, weist am rechten Oberlide ein ausgedehntes Kolobom auf. Der durchgreifende Defekt nimmt fast die ganze innere Hälfte des rechten Oberlides ein und erstreckt sich bis in $\mathrm{d}^{3} / 8 \mathrm{n}$ Lidwinkel, wo die ver, größerte Karunkel in einem stehengebliebenen Hautstück aufgegangen ist. Vom oberen bogen-förmigen Rande des Koloboms zieht ein Hautschaltstück auf kürzestem Wege zum Bulbus, wo es adhärent ist. Ein Fornix fehlt an dieser Stella und ebenso fehl·t das obere Tränenröhrchen. Während des Schlafens is $\notin$ ein schmaler Teil der medialen Hornhauthälfte unbedeckt. Da sich erfahrungsgemäß die meÌ9ten Individuen mit angeborenem Lidkolobom als größere Kinder bereits mit schweren dauernden Veränderungen der Hornhaut nach Keratitis e lagophthalmo vorstellen, so muß zu frühzeitiger opera $<$ tiver Beseitigung eines angeborenen Lidkoloboms geraten werden.

Balcarek: Demonstration von Flocculi der Iris.

Politzer; Über den Einíluß der Röntgenstrahlen auf die embryonale Linse.

Meine Herrenl Daß Röntgenstrahlen Schädigungen der Linse her-vorrufen können, ist einwandfrei bewiesen. Fraglich ist jedoch, ob den Röntgenstrahlen eine besondere, für Röntgenstrahlen charakteristische Form der Linsenschädigung entspricht. Fraglich ist ferner, auf welche Art und Weise diese Schädigung der Linse zustande kommt, endlich, nach welchen Strahlenmengen wir den Eintritt eines solchen Ereignisseis gewärtigen müssen.

Ehe ich zu einer Besprechung des einschlägigen Schrifttums über-gehe, seien die Ergebnisse einer Versuchsreihe kurz besprochen, welche ich vor einiger Zeit ausgeführt habe. Als Versuchsmaterial dienten Larven von Salamandra maculosa, welche einer Totalbestrahlung unterworfen worden waren. Die hier geschilderten Veränderungen traten bei Strahlenmengen über 8 H/i A regelmäßig ein. Die Larven (insgesamt über 100)

Ophthalmologische Gesellschaft in Wien.

305 wurden zu verschiedenen Zeitpunkten nach der Bestrahlung in Bourns Gemisch fixiert und in lückenlose Schnittreihen zerlegt. Die Schnit1/18e wurden mit Hämatoxylin und Eosin gefärbt. In den ersten zwei Monaten nach der Bestrahlung sind - abgesehen von den noch zu schildernden abnormen Karyokinesen - keine patho-logischen Veränderungen des Auges nachweisbar. Im dritten Monate schwillt die Linse an; sie erhält eine höckerige Oberfläche. Dann reißt die Linsenkapsel ein, worauf Linsenfasermasse in die Augenkammer aus-tritt. Die Linsenfasern quellen auf und werden im vierten Monate nach der Bestrahlung resorbiert. Zu Ende des vierten Monates sind keine Reste der Linse mehr nachweisbar.

Diese Veränderungen könnten nun in der folgenden Weise erklärt werden. Duirch die Bestrahlung erfährt die Linsenkapsel eine Verände-rung ihrer Durchlässigkeit. Das Kammerwasser dringt in die Linse ein und bringt die Fasern zur Verquellung. Dadurch nimmt die Linse an Größe zu. Die Linsenkapsel reißt ein und die Linsenfasern treten in die 
Augenkammer aus, wo sie resorbiert werden. Für diese Deutung unserer Versuchsergebnisse spricht der Umstand, daß tatsächlich eine Er-höhung der Durchlässigkeit organismischer und anorganismischer Membranen nach Röntgenbestrahlung beobachtet und zur Erklärung bestimmter kli-nischer Bestrahlungseffekte (Früherythem, Inkretstoß der Thyreoidea bei Basedowscher Krankheit) mit Erfolg verwertet wurde. Hingegen treten alle diese vermutlich durch Erhöhung der Membranpermeabilität be-dingten Effekte unmittelbar oder wenige Stunden nach der Bestrahlung auf, während der Untergang der Linse in unseren Versuchen erst zu Ende des dritten Monates beginnt.

Vielleicht trifft der nachfolgende Erklärungsversuch das Richtige: Die Röntgenstrahlen bewirken Veränderungen der Karyokinese. Kurz nach der Bestrahlung treten in den bestrahlten Geweben abnorme Zellteilungen (Pyknosen und Pseudoamitosen) auf. Die Zahl der Zellteilungen nimmt rasch ab; wenige Stunden nach der Bestrahlung ist der „Primär-meffekt” abgeklungen und die Gewebe sind mitosenfrei. Diese ,,mitosenfreie” Zwischenzeit dauert einige Tage; dann treten neuerdings Karyokinesen auf (Sekund $1 / 8$ reffekt), welche gleichfalls abnorm sind, sich aber von denen des Primäreffektes deutlich unterscheiden. Die Chromosomen sind ver-schieden lang; zum Teil sind sie in kleine Bruchstücke zerfallen. Nicht alle Chromosomen gelangen zu den Polen der Teilungsspindel. Einige werden vielmehr an die Peripherie der Zelle abgelenkt. Da diese abgelenkten Gebilde gleichzeitig mit den zu den Polen gelangten Chromosomen in die weiteren Teilungsphasen eintreten, entsфehen Zellen, welche neben dem aus den zu den Polen gelangten Chromosomen gebildeten Kerne noch einen oder mehrere aus den abgelenkten Chromosomen und Chromosomenbruchstücken hervorgegangene „Teilkerne” besitzen. Nach Verabreichung sehr großer Strahlendosen ist oftmals die Schädigung der Chromosomen so stark, daß Tochtersterne überhaupt nicht gebildet werden, sondern die Zelle vielmehr aus der Mitose direkt in Nekrose übergeht.

Tatsächlich finden sich nun in dem Linsenepithel unserer Versuchs-tiere pathologische Karyokinesen, welche zum Untergang zahlreicher Zellen führen. Im dritten Monate nach der Bestrahlung sind nur mehr

$20 * *$

306 Ophthalmologische Gesellschaft in Wien.

spärliche Reste eines Linsenepithels vorhanden. In diesem Zeitpunkte setzt nun die Verquellung und der Untergang der Linse ein. Es er-scheint somit die Annahme berechtigt, daß durch den Untergang des Linsenepithels Bedingungen gcschaffen werden, welche ein Eindringen des Kammerwassets in die Linse ermöglichen. Dadurch kommt es zur Verquellung der Linsen†jasem, zur Größenzunahme der Linse überhaupt und (wie bereits oben ausgeführt) zur Berstung der Linsenkapsel.

Diese Versuchsergebnisse lassen sich selbstverständlich nicht ohne weiteres auf das Gebiet der Röntgentherapie am Menschen übertragen. Vor allem ist die embryonale Linse und die Linse neugeborener Tiere, wie aus den Versuchen von Tribondeau und seinen Schülern hervorgeht, viel empfindlicher als die Linse erwachsener Tiere. Ferner liegen Salamander und Mensch in der Tierreihe so weit auseinander, daß die Versuchsergebnisse an Salamanderlarven natürlich keine Voraussage bestimm-ter therapeutischer Effekte der Róntgenstrahlen beim Menschen gestatten. Die im Schrifttum niedergelegten Untersuchungen über die Rònt-genempfindlichkeit der Säugetierlinse führen aber zu sehr widerspre-chenden Ergebnissen. Während Rados und Schinz glauben, die Un-schädlichkeit selbst sehr großer Strahlenmengen auf das Auge des er-wachsenen Kaninchens erwiesen zu haben, kornmt Rohrschneíder auf Grund von Versuchen an dem 
gleichen Materiale zu dem Ergebnisse, daß bereits 2 H E D regelmäßig Trübung der Linse bewirken.

Vom therapeutischen Standpunkte ware aus all dem vorläufig fob gender Arbeitsplan abzuleiten: Starkbestrahlung des Auges kommt nur bei malignen Erkrankungen des Auges in Frage. Bei der Starkbestrahlung endokranieller Prozesse ist das Auge womöglich aus dem direkten Durchstrahlungsbereich auszuschalten. Diese Vorsichtsmaßnahme ist ge-boten, obwohl es zweifellos Fälle gibt, bei welchen trotz Starkbestrahlung des Auges keine Linsentrübungen auftraten. Die Schwachbestrahlungen, welche bei entziindlichen Augenerkrankungen verabfolgt werden, sind sicher unschädlich.

Augenärztliche Gesellschaft in Hamburg.

Avis den Sitzungen des Winter semesters 1928-29.

O. Beselin zeigt einen 78jährigen Patienten mit gürtelförmiger Horn-hauttrübung bds. mit gutem Sehvermögen, rechts S. 4/4, links 4/7. Sie verdeckte nur die untere Hälfte der Pupillen und verursachte eine Be-lästigung erst dann, als sich in der Miite im linken Auge oberflächliche Teile abgestoßen hatten und hierdurch Schmerzen verursacht wurden. Durch Abschabung wurden die Beschwerden beseitigt. Das getrübte Ge-webe ließ sich in einem Bezirk von $2 \mathrm{~mm}$ Durchmesser wie eine Kruste abheben. Es entstand hierdurch in der Trübung eine klare Stelle mit zackiger Begrenzung, in der das neugebildete Epithel, die Eowmansche Membran und die Hornhautlamellen ungetrübt und durchsichtig waren.

D. Derselbe berichtet von einem Fall von syphilitischer Gefäßneubil-dung von der Papille in den Glaskörper. Eine 36jährige Patientin hatte bei frischer Sehstörung weiße Einscheidung mehrerer Netzhautarterien ein venöser Gefäßknäuel auf der Papille viele Netzhaut- und spärliche 